PROCEEDINGS OF THE

AMERICAN MATHEMATICAL SOCIETY

Volume 135, Number 9, September 2007, Pages 2877-2886

S 0002-9939(07)08803-X

Article electronically published on May 8, 2007

\title{
THE CHARACTERISTIC FUNCTION OF A COMPLEX SYMMETRIC CONTRACTION
}

\author{
NICOLAS CHEVROT, EMMANUEL FRICAIN, AND DAN TIMOTIN
}

(Communicated by Joseph A. Ball)

\begin{abstract}
It is shown that a contraction on a Hilbert space is complex symmetric if and only if the values of its characteristic function are all symmetric with respect to a fixed conjugation. Applications are given to the description of complex symmetric contractions with defect indices equal to 2 .
\end{abstract}

\section{INTRODUCTION}

Complex symmetric operators on a complex Hilbert space are characterized by the existence of an orthonormal basis with respect to which their matrix is symmetric. Their theory is therefore connected with the theory of symmetric matrices, which is a classical topic in linear algebra. A more intrinsic definition implies the introduction of a conjugation in the Hilbert space, that is, a conjugate-linear, isometric and involutive map, with respect to which the symmetry is defined. Such operators or matrices appear naturally in many different areas of mathematics and physics; we refer to [5] for more about the history of the subject and its connections to other domains, as well as for an extended list of references.

The interest in complex symmetric operators has been recently revived by the work of Garcia and Putinar [3, 4, 5]. In their papers a general framework is established for such operators, and it is shown that large classes of operators on a Hilbert space can be studied in this framework. The examples are rather diverse: normal operators are complex symmetric, for instance, but also certain types of Volterra and Toeplitz operators, as well as the so-called compressed shift on the functional model spaces $H^{2} \ominus \phi H^{2}$, where $\phi$ denotes a nonconstant inner function.

The purpose of this paper is to explore further the generalizations of this last example. The natural context is the model theory of completely nonunitary contractions developed by Sz. Nagy and Foias [6]. The main result is a criterion for a contraction to be complex symmetric in terms of its characteristic function. In the sequel some applications of this result are given.

The plan of the paper is the following. The next section presents preliminary material. Section 3 contains the announced criterion. In Section 4 one discusses $2 \times 2$ inner characteristic functions, and the results are applied in the last section in order to obtain a series of examples of complex symmetric contractions with defect indices 2 .

Received by the editors April 8, 2006 and, in revised form, May 28, 2006.

2000 Mathematics Subject Classification. Primary 47A45, 47B15.

Key words and phrases. Complex symmetric operator, contraction, characteristic function. 


\section{Preliminaries}

2.1. Complex symmetric operators. We first recall some basic facts from [3, 4, 5. Let $\mathcal{H}$ be a complex Hilbert space, and $\mathcal{L}(\mathcal{H})$ the algebra of all bounded linear operators on $\mathcal{H}$. A conjugation $C$ on $\mathcal{H}$ is a conjugate-linear, isometric and involutive map; thus $C^{2}=I$, and $\langle C f, C g\rangle=\langle g, f\rangle$ for all $f, g \in \mathcal{H}$.

For a fixed conjugation operator $C$ on $\mathcal{H}$, we say that a linear operator $T$ on $\mathcal{H}$ is $C$-symmetric if $T=C T^{*} C$. One sees immediately that if $T$ is $C$-symmetric, then $T^{*}$ is $C$-symmetric. Then $T \in \mathcal{L}(\mathcal{H})$ is called complex symmetric if there exists a conjugation $C$ on $\mathcal{H}$ such that $T$ is $C$-symmetric. Among various examples of complex symmetric operators [5], we mention the class of normal operators; in particular, unitary operators are complex symmetric. Also, direct sums of complex symmetric operators are complex symmetric.

Complex symmetric operators can also be characterized in terms of certain matrix representations, as shown by the following result from [5].

Lemma 2.1. Let $C$ be a conjugation on $\mathcal{H}$. Then:

(i) There exists an orthonormal basis $\left(e_{n}\right)_{n=1}^{\operatorname{dim} \mathcal{H}}$ of $\mathcal{H}$ such that $C e_{n}=e_{n}$ for all $n$; such a basis is called a $C$-real orthonormal basis for $\mathcal{H}$.

(ii) $T \in \mathcal{L}(\mathcal{H})$ is $C$-symmetric if and only if there exists a $C$-real orthonormal basis $\left(e_{n}\right)_{n=1}^{\operatorname{dim} \mathcal{H}}$ for $\mathcal{H}$ such that

$$
\left\langle T e_{n}, e_{m}\right\rangle=\left\langle T e_{m}, e_{n}\right\rangle, \quad \forall n, m \geq 1 .
$$

2.2. Characteristic functions and model operators. The characteristic function for a contraction and the construction of the basic functional model is developed by B. Sz.-Nagy and C. Foias [6], which is the main source for this subsection. In the sequel $\mathbb{D}$ and $\mathbb{T}$ denote the unit disc and the unit circle in the complex plane.

Suppose $T \in \mathcal{L}(\mathcal{H}),\|T\| \leq 1$. There is a unique decomposition $\mathcal{H}=\mathcal{H}_{0} \oplus \mathcal{H}_{u}$ such that $T \mathcal{H}_{0} \subset \mathcal{H}_{0}, T \mathcal{H}_{u} \subset \mathcal{H}_{u}$ and $T_{\mid \mathcal{H}_{u}}$ is unitary, whereas $T_{\mid \mathcal{H}_{0}}$ is completely nonunitary (c.n.u.), that is, $T_{\mid \mathcal{H}_{0}}$ is not unitary on any of its invariant subspaces.

The operator $D_{T}=\left(I-T^{*} T\right)^{1 / 2}$ is called the defect operator of $T$. The defect spaces of $T$ are $\mathcal{D}_{T}=\overline{D_{T} \mathcal{H}}, \mathcal{D}_{T^{*}}=\overline{D_{T^{*}} \mathcal{H}}$, and the defect indices $\partial_{T}=\operatorname{dim} \mathcal{D}_{T}$, $\partial_{T^{*}}=\operatorname{dim} \mathcal{D}_{T^{*}}$. Since $D_{T}=D_{T_{0}} \oplus 0, D_{T^{*}}=D_{T_{0}^{*}} \oplus 0$, we have $\mathcal{D}_{T}=\mathcal{D}_{T_{0}}$ and $\mathcal{D}_{T^{*}}=\mathcal{D}_{T_{0}^{*}}$.

We say that $T \in C_{0}$. if $T^{n} \rightarrow 0$ strongly, and $T \in C_{0}$ if $T^{*} \in C_{0}$; also, $C_{00}=C_{0} \cdot \cap C_{\cdot 0}$.

Suppose $\mathcal{E}, \mathcal{E}^{\prime}$ are Hilbert spaces, and $\Theta: \mathbb{D} \rightarrow \mathcal{L}\left(\mathcal{E}, \mathcal{E}^{\prime}\right)$ is a contraction-valued analytic function. One can decompose $\mathcal{E}=\mathcal{E}_{p} \oplus \mathcal{E}_{u}, \mathcal{E}^{\prime}=\mathcal{E}_{p}^{\prime} \oplus \mathcal{E}_{u}^{\prime}$, such that:

- for all $z \in \mathbb{D}, \Theta(z) \mathcal{E}_{p} \subset \mathcal{E}_{p}^{\prime}, \Theta(z) \mathcal{E}_{u} \subset \mathcal{E}_{u}^{\prime}$;

- if $\Theta=\Theta_{p} \oplus \Theta_{u}$ is the corresponding decomposition of $\Theta$, then $\Theta_{p}$ is pure, that is, $\left\|\Theta_{p}(0) h\right\|<\|h\|$ for all $h \in \mathcal{E}_{p}, h \neq 0$, while $\Theta_{u}$ is a unitary constant.

$\Theta_{p}$ is then called the pure part of $\Theta$.

One says [6] that two contractive analytic functions $\Theta: \mathbb{D} \rightarrow \mathcal{L}\left(\mathcal{E}, \mathcal{E}_{*}\right), \Theta^{\prime}$ : $\mathbb{D} \rightarrow \mathcal{L}\left(\mathcal{E}^{\prime}, \mathcal{E}_{*}^{\prime}\right)$ coincide if there are unitaries $U: \mathcal{E} \rightarrow \mathcal{E}^{\prime}, U_{*}: \mathcal{E}_{*} \rightarrow \mathcal{E}_{*}^{\prime}$, such that $\Theta(z)=U_{*}^{*} \Theta^{\prime}(z) U$ for all $z \in \mathbb{D}$.

The characteristic function of $T$ is an operator valued function $\Theta_{T}(\lambda): \mathcal{D}_{T} \rightarrow$ $\mathcal{D}_{T^{*}}$ defined for $\lambda \in \mathbb{D}$ by

$$
\Theta_{T}(\lambda):=-T+\lambda D_{T^{*}}\left(I-\lambda T^{*}\right)^{-1} D_{T} \mid \mathcal{D}_{T} .
$$


$\Theta_{T}$ is a pure contraction-valued analytic function on $\mathbb{D}$, and one easily sees that $\Theta_{T}=\Theta_{T_{0}}$.

For $\mathcal{E}$ a Hilbert space, we denote by $L^{2}(\mathcal{E})$ the Lebesgue space of measurable functions $f: \mathbb{T} \rightarrow \mathcal{E}$ of square integrable norm, and by $H^{2}(\mathcal{E}) \subset L^{2}(\mathcal{E})$ the Hardy space of functions whose negative Fourier coefficients vanish. $P_{+}$is the orthogonal projection onto $H^{2}(\mathcal{E})$, and $P_{-}=I-P_{+}$.

If we are given an arbitrary contraction-valued analytic function $\Theta: \mathbb{D} \rightarrow$ $\mathcal{L}\left(\mathcal{E}, \mathcal{E}^{\prime}\right)\left(\mathcal{E}, \mathcal{E}_{*}\right.$ Hilbert spaces $)$, one defines the model space associated to $\Theta$ by

$$
\mathfrak{K}_{\Theta}=\left(H^{2}\left(\mathcal{E}_{*}\right) \oplus \overline{\left(I-\Theta^{*} \Theta\right)^{1 / 2} L^{2}(\mathcal{E})}\right) \ominus\left\{\Theta_{T} f \oplus\left(I-\Theta^{*} \Theta\right)^{1 / 2} f: f \in H^{2}(\mathcal{E})\right\},
$$

and the model operator $\mathbf{T}_{\Theta} \in \mathcal{L}\left(\mathfrak{K}_{\Theta}\right)$ by

$$
\mathbf{T}_{\Theta}(f \oplus g)=P_{\mathfrak{K}_{\Theta}}(z f \oplus z g)
$$

$\left(P_{\mathfrak{K}_{\Theta}}\right.$ is the orthogonal projection onto $\left.\mathfrak{K}_{\Theta}\right)$. Then $\mathbf{T}_{\Theta}$ is a c.n.u. contraction, and its characteristic function coincides with the pure part of $\Theta$.

If we start with a contraction $T$, and apply the previous constructions to $\Theta_{T}$, the resulting operator $\mathbf{T}_{\Theta_{T}}$ is unitarily equivalent to $T_{0}$ (the completely non-unitary part of $T$ ).

A contractive analytic function $\Theta$ is called inner if its boundary values $\Theta\left(e^{i t}\right)$ are isometries a.e. on $\mathbb{T}$. If $T$ is c.n.u., then $T \in C_{.0}$ if and only if $\Theta_{T}$ is inner.

\section{The MAIN THEOREM}

Our main result gives a criterion for complex symmetric contractions.

Theorem 3.1. Let $T$ be a contraction on the Hilbert space $\mathcal{H}$. Then the following are equivalent:

(i) $T$ is complex symmetric.

(ii) There exists a conjugate-linear map $J: \mathcal{D}_{T} \rightarrow \mathcal{D}_{T^{*}}$ which is isometric, onto and satisfies

$$
\Theta_{T}(z)=J \Theta_{T}(z)^{*} J, \quad \forall z \in \mathbb{D} .
$$

(iii) There exists a Hilbert space $\mathcal{E}$, a conjugation $J^{\prime}$ on $\mathcal{E}$, and a pure contractive analytic function $\Theta: \mathbb{D} \rightarrow \mathcal{L}(\mathcal{E})$, whose values are $J^{\prime}$-symmetric operators, such that $\Theta_{T}$ coincides with $\Theta$.

Proof. (i) $\Rightarrow$ (ii) If $T$ is complex symmetric, there exists a conjugation $C$ on $\mathcal{H}$ such that $T=C T^{*} C$. Since $C$ is involutive, we get $C T^{*}=T C, C T=T^{*} C$, and $C\left(I-T^{*} T\right)=\left(I-T T^{*}\right) C$. Thus $C D_{T}^{2}=D_{T^{*}}^{2} C$, and therefore $C D_{T}^{2 n}=D_{T^{*}}^{2 n} C$, $n \geq 0$. If $\left(p_{n}\right)_{n \geq 1}$ is a sequence of polynomials tending uniformly to $\sqrt{x}$ on $[0,1]$, then $C p_{n}\left(D_{T}^{2}\right)=p_{n}\left(D_{T^{*}}^{2}\right) C$, whence $C D_{T}=D_{T^{*}} C$. In particular, $C \mathcal{D}_{T} \subset \mathcal{D}_{T^{*}}$; since $T^{*}$ is also $C$-symmetric, we actually have equality. Moreover, $C T^{n}=T^{* n} C$ for all $n \geq 1$ implies $C(I-\bar{z} T)^{-1}=\left(I-z T^{*}\right)^{-1} C$.

Now define $J:=C \mid \mathcal{D}_{T}$. Then $J$ is a conjugate-linear map from $\mathcal{D}_{T}$ onto $\mathcal{D}_{T^{*}}$ which is isometric, and the equalities above imply that $J \Theta_{T}(z)^{*} J=\Theta_{T}(z)$ for all $z \in \mathbb{D}$.

(ii) $\Rightarrow$ (i) Assume first that $T$ is completely nonunitary. We will prove that the model operator $\mathbf{T}_{\Theta_{T}} \in \mathcal{L}\left(\mathfrak{K}_{\Theta_{T}}\right)$, as defined by (2.2) and (2.3), is complex symmetric. For simplicity, we will write in the sequel of the proof $\mathbf{T}$ and $\mathfrak{K}$ instead of $\mathbf{T}_{\Theta_{T}}$ and $\mathfrak{K}_{\Theta_{T}}$. 
Let us introduce some supplementary notation. Define

$$
\mathfrak{H}:=L^{2}\left(\mathcal{D}_{T^{*}}\right) \oplus \overline{\left(I-\Theta_{T}^{*} \Theta_{T}\right)^{1 / 2} L^{2}\left(\mathcal{D}_{T}\right)}
$$

and $\pi: L^{2}\left(\mathcal{D}_{T}\right) \rightarrow \mathfrak{H}, \pi_{*}: L^{2}\left(\mathcal{D}_{T^{*}}\right) \rightarrow \mathfrak{H}$ by

$$
\pi(f)=\Theta_{T} f \oplus\left(I-\Theta_{T}^{*} \Theta_{T}\right)^{1 / 2} f, \quad \pi_{*}(g)=g \oplus 0,
$$

for $f \in L^{2}\left(\mathcal{D}_{T}\right)$ and $g \in L^{2}\left(\mathcal{D}_{T^{*}}\right)$. Then $\pi$ and $\pi_{*}$ are isometries, $\mathfrak{H}$ is spanned by $\pi L^{2}\left(\mathcal{D}_{T}\right)$ and $\pi_{*} L^{2}\left(\mathcal{D}_{T^{*}}\right), \pi_{*}^{*} \pi=\Theta_{T}$, and $\mathfrak{K}=\mathfrak{H} \ominus\left(\pi H^{2}\left(\mathcal{D}_{T}\right) \oplus \pi_{*} H_{-}^{2}\left(\mathcal{D}_{T^{*}}\right)\right)$. If $P$ denotes the orthogonal projection (in $\mathfrak{H}$ ) onto $\mathfrak{K}$, then $P=I_{\mathfrak{H}}-\pi P_{+} \pi^{*}-\pi_{*} P_{-} \pi_{*}^{*}$.

Let $Z \in \mathcal{L}(\mathfrak{H})$ be the unitary operator which acts as multiplication by $z$ on both coordinates. Then $\pi(z f)=Z \pi f, \pi_{*}(z g)=Z \pi_{*} g$, and, according to (2.3), $\mathbf{T}=P Z \mid \mathfrak{K}$.

If $\widetilde{J}: L^{2}\left(\mathcal{D}_{T}\right) \rightarrow L^{2}\left(\mathcal{D}_{T^{*}}\right)$ is defined by $(\widetilde{J} f)(z)=\bar{z} J(f(z))$, then $\widetilde{J}$ is conjugatelinear, isometric and onto; moreover

$$
\widetilde{J} P_{+}=P_{-} \widetilde{J}, \quad \widetilde{J} H^{2}\left(\mathcal{D}_{T}\right)=H_{-}^{2}\left(\mathcal{D}_{T^{*}}\right),
$$

and $\widetilde{J}^{-1} g(z)=\bar{z} J^{-1} g(z)$ for $g \in L^{2}\left(\mathcal{D}_{T^{*}}\right)$.

We define the conjugate-linear map $C: \mathfrak{H} \rightarrow \mathfrak{H}$ by the formula

$$
C\left(\pi f+\pi_{*} g\right):=\pi_{*}(\widetilde{J} f)+\pi\left(\widetilde{J}^{-1} g\right), \quad f \in L^{2}\left(\mathcal{D}_{T}\right), g \in L^{2}\left(\mathcal{D}_{T^{*}}\right) .
$$

We prove first that $C$ is a conjugation on $\mathfrak{H}$ and that $Z$ is $C$-symmetric. Since $\pi, \pi_{*}, \widetilde{J}, \widetilde{J}^{-1}$ are (linear or conjugate-linear) isometries, it follows that for all $f, h \in$ $L^{2}\left(\mathcal{D}_{T}\right)$ and all $g, k \in L^{2}\left(\mathcal{D}_{T^{*}}\right)$,

$$
\begin{aligned}
&\left\langle C\left(\pi f+\pi_{*} g\right), C\left(\pi h+\pi_{*} k\right)\right\rangle=\left\langle\pi\left(\widetilde{J}^{-1} g\right), \pi\left(\widetilde{J}^{-1} k\right)\right\rangle+\left\langle\pi_{*}(\widetilde{J} f), \pi_{*}(\widetilde{J} h)\right\rangle \\
&+\left\langle\pi\left(\widetilde{J}^{-1} g\right), \pi_{*}(\widetilde{J} h)\right\rangle+\left\langle\pi_{*}(\widetilde{J} f), \pi\left(\widetilde{J}^{-1} k\right)\right\rangle \\
&=\langle k, g\rangle+\langle h, f\rangle+\left\langle\Theta_{T} \widetilde{J}^{-1} g, \widetilde{J} h\right\rangle+\left\langle\widetilde{J} f, \Theta_{T} \widetilde{J}^{-1} k\right\rangle .
\end{aligned}
$$

But $J \Theta_{T}(z)^{*} J=\Theta_{T}(z)$ implies $\Theta_{T} \widetilde{J}^{-1}=\widetilde{J} \Theta_{T}^{*}$, and therefore

$$
\begin{aligned}
\left\langle C\left(\pi f+\pi_{*} g\right), C\left(\pi h+\pi_{*} k\right)\right\rangle & =\langle k, g\rangle+\langle h, f\rangle+\left\langle\widetilde{J} \Theta_{T}^{*} g, \widetilde{J} h\right\rangle+\left\langle\widetilde{J} f, \widetilde{J} \Theta_{T}^{*} k\right\rangle \\
& =\langle k, g\rangle+\langle h, f\rangle+\left\langle h, \Theta_{T}^{*} g\right\rangle+\left\langle\Theta_{T}^{*} k, f\right\rangle \\
& =\langle k, g\rangle+\langle h, f\rangle+\left\langle h, \pi^{*} \pi_{*} g\right\rangle+\left\langle\pi^{*} \pi_{*} k, f\right\rangle \\
& =\left\langle\pi h+\pi_{*} k, \pi f+\pi_{*} g\right\rangle .
\end{aligned}
$$

Thus $C$ is a well-defined isometric conjugate-linear map. It follows immediately from the definition that $C^{2}=I_{\mathfrak{H}}$ and thus $C$ is a conjugation on $\mathfrak{H}$.

If $f \in L^{2}\left(\mathcal{D}_{T}\right)$, then

$$
\begin{aligned}
C Z C(\pi(f)) & =C Z \pi_{*}(\widetilde{J} f)=C \pi_{*}(z \widetilde{J} f)=C \pi_{*}(J f) \\
& =\pi\left(\widetilde{J}^{-1} J f\right)=\pi\left(\bar{z} J^{-1} J f\right)=\pi(\bar{z} f)=Z^{*} \pi(f) .
\end{aligned}
$$

Similarly one proves that $C Z C\left(\pi_{*}(g)\right)=Z^{*} \pi_{*}(g)$ for $g \in L^{2}\left(\mathcal{D}_{T^{*}}\right)$, and therefore $C Z C=Z^{*}$; that is, $Z$ is $C$-symmetric.

By (3.2), $C\left(\pi H^{2}\left(\mathcal{D}_{T}\right)\right)=\pi_{*} \widetilde{J} H^{2}\left(\mathcal{D}_{T}\right)=\pi_{*} H_{-}^{2}\left(\mathcal{D}_{T^{*}}\right)$ and $C\left(\pi_{*} H_{-}^{2}\left(\mathcal{D}_{T^{*}}\right)\right)=$ $\pi H^{2}\left(\mathcal{D}_{T}\right)$. Since $C$ is isometric, we have

$$
C \mathfrak{K}=C \mathfrak{H} \ominus C\left(\pi H^{2}\left(\mathcal{D}_{T}\right) \oplus \pi_{*} H_{-}^{2}\left(\mathcal{D}_{T^{*}}\right)\right)=\mathfrak{H} \ominus\left(\pi H^{2}\left(\mathcal{D}_{T}\right) \oplus \pi_{*} H_{-}^{2}\left(\mathcal{D}_{T^{*}}\right)\right)=\mathfrak{K} .
$$


Therefore the restriction $C^{\prime}$ of $C$ to $\mathfrak{K}$ is a conjugation on $\mathfrak{K}$. Since $C$ leaves $\mathfrak{K}$ and its orthogonal invariant, we have $C|\mathfrak{K}=P C P| \mathfrak{K}$ and $P C\left(I_{\mathfrak{H}}-P\right)=0$. Therefore

$$
\mathbf{T}=P Z\left|\mathfrak{K}=P C Z^{*} C\right| \mathfrak{K}=P C P Z^{*} P C P \mid \mathfrak{K}=C^{\prime} \mathbf{T}^{*} C^{\prime} .
$$

Thus $\mathbf{T}$ is $C^{\prime}$-symmetric. Since $T$ is completely nonunitary, $T$ is unitarily equivalent to $\mathbf{T}$ and is therefore also complex symmetric.

Now, let $T \in \mathcal{L}(\mathcal{H})$ be a general contraction satisfying condition (ii) in the statement of the theorem. If we decompose $T=T_{0} \oplus T_{u}$, with $T_{0}$ c.n.u. and $T_{u}$ unitary, then $T_{0}$ also satisfies (ii), and it is therefore complex symmetric by the above argument. Since $T_{u}$ is unitary, it is complex symmetric. Therefore $T$, being the direct sum of two complex symmetric operators, is also complex symmetric.

(ii) $\Rightarrow$ (iii) If (3.1) is satisfied, and $C^{\prime}$ is some conjugation on $\mathcal{D}_{T}$, then $U=$ $J C^{\prime}: \mathcal{D}_{T} \rightarrow \mathcal{D}_{T^{*}}$ is unitary and $C^{\prime}=U^{*} J$. If $\Theta: \mathbb{D} \rightarrow \mathcal{L}\left(\mathcal{D}_{T}\right)$ is defined by $\Theta(z)=U^{*} \Theta_{T}(z)$, then

$$
\Theta(z)=U^{*} J \Theta_{T}(z)^{*} J=U^{*} J\left(U^{*} \Theta_{T}(z)\right)^{*} U^{*} J=C^{\prime} \Theta(z)^{*} C^{\prime} .
$$

(iii) $\Rightarrow$ (ii) If $U: \mathcal{E} \rightarrow \mathcal{D}_{T}, U_{*}: \mathcal{E} \rightarrow \mathcal{D}_{T^{*}}$ are unitary operators satisfying $\Theta_{T}(z)=U_{*} \Theta(z) U^{*}$ for all $z \in \mathbb{D}$, then $J=U_{*} J^{\prime} U^{*}$ satisfies all requirements in (ii).

Corollary 3.2. A contraction $T$ with $\partial_{T}=\partial_{T^{*}}=1$ is complex symmetric.

Proof. If $\partial_{T}=\partial_{T^{*}}=1$, then $\Theta_{T}$ is scalar-valued, and we may identify $\mathcal{D}_{T}$ and $\mathcal{D}_{T^{*}}$ with $\mathbb{C}$. The natural conjugation $J$ on $\mathbb{C}$ defined by $J(z)=\bar{z}$ then satisfies condition (iii) in Theorem 3.1, whence $T$ is complex symmetric.

For the case $T \in C_{00}$, Corollary 3.2 is proved in [5] and [4], where more of its consequences are developed. Also in [5] one can find the next result, for which we give a different proof.

Corollary 3.3. Any operator on a 2-dimensional space is complex symmetric.

Proof. Since the complex symmetry is preserved by multiplication with nonzero scalars, it is enough to assume $\|T\|=1$. But then either $T$ is unitary, or $\partial_{T}=$ $\partial_{T^{*}}=1$, in which case we may apply Corollary 3.2 .

It follows from Theorem 3.1 that if a contraction $T$ is complex symmetric, then $\partial_{T}=\partial_{T^{*}}$. However, this is also a consequence of a more general result from [5], namely that if a (not necessarily contractive) operator $T$ is complex symmetric, then $\operatorname{dim} \operatorname{ker} T=\operatorname{dim} \operatorname{ker} T^{*}$.

\section{4. $2 \times 2$ INNER FUNCTIONS}

As shown in Corollary 3.2, contractions with defect indices 1 are always complex symmetric. As an application of Theorem 3.1, we will discuss in this section the case $\partial_{T}=\partial_{T^{*}}=2$. We assume moreover that the characteristic function $\Theta_{T}$ is inner, which is equivalent to $T \in C_{00}$.

Definition 4.1. Let $\Theta: \mathbb{D} \rightarrow \mathcal{L}\left(\mathcal{E}, \mathcal{E}_{*}\right)$ be a contractive analytic function. We say that $\Theta$ is symmetrizable if its matrix with respect to some fixed orthonormal bases (independent of $z$ ) in $\mathcal{E}$ and $\mathcal{E}_{*}$ is symmetric for all $z \in \mathbb{D}$. 
According to Lemma 2.1 and Theorem 3.1. (iii), a contraction is complex symmetric if and only if its characteristic function is symmetrizable. We are interested in this section in $2 \times 2$ matrix-valued characteristic functions $\Theta(z)$. Note that Corollary 3.3 implies that, for all $z \in \mathbb{D}$, there exist $U_{1}(z), U_{2}(z)$ unitary such that $U_{1}(z) \Theta(z) U_{2}(z)$ is symmetric. But, in order to find symmetrizable analytic functions, the matrices $U_{1}$ and $U_{2}$ should not depend on $z$.

We recall the following result in 3 which gives a parametrization of $2 \times 2$ inner functions.

Proposition 4.2. Suppose $\phi$ is a nonconstant inner function in $H^{\infty}, a, b, c, d \in$ $H^{\infty}$, and

$$
\Theta(z)=\left(\begin{array}{cc}
a(z) & -b(z) \\
c(z) & d(z)
\end{array}\right)
$$

Then $\Theta$ is a $2 \times 2$ inner function and det $\Theta=\phi$ if and only if

(i) $a, b, c, d$ belong to $\mathcal{H}(z \phi)=H^{2} \ominus z \phi H^{2}$;

(ii) $d=C(a)$ and $c=C(b)$;

(iii) $|a|^{2}+|b|^{2}=1$ a.e. on $\mathbb{T}$.

Here $C$ denotes the natural conjugation on $\mathcal{H}_{z \phi}$ defined by

$$
C(f)=\bar{f} \phi \quad\left(f \in \mathcal{H}_{z \phi}\right) .
$$

The following result characterizes the symmetrizable $2 \times 2$ matrix-valued inner functions.

Theorem 4.3. A $2 \times 2$ inner function $\Theta(z)=\left(\begin{array}{cc}a(z) & -b(z) \\ C(b)(z) & C(a)(z)\end{array}\right)$ is symmetrizable if and only if there exists $(\gamma, \theta) \neq(0,0)$ such that $\gamma a+\theta b$ is a fixed point of $C$, where $C$ is defined by (4.1), $\phi=\operatorname{det} \Theta$.

Proof. Suppose there exists $(\gamma, \theta) \neq(0,0)$ such that $C(\gamma a+\theta b)=\gamma a+\theta b$; we may assume that $|\gamma|^{2}+|\theta|^{2}=1$. Define the unitary matrix $U$ by $U=\left(\begin{array}{cc}\bar{\theta}-\gamma \\ \bar{\gamma} & \theta\end{array}\right)$. Then

$$
\left(\begin{array}{cc}
-i & 0 \\
0 & i
\end{array}\right) \Theta(z) U=\left(\begin{array}{cc}
-i \bar{\theta} a(z)+i \bar{\gamma} b(z) & i(\gamma a(z)+\theta b(z)) \\
i(\gamma a(z)+\theta b(z)) & -i \gamma C(b)(z)+i \theta C(a)(z)
\end{array}\right)
$$

since $\bar{\theta} C(b)(z)+\bar{\gamma} C(a)(z)=C(\gamma a+\theta b)(z)=(\gamma a+\theta b)(z)$. Therefore $\Theta$ is symmetrizable.

Reciprocally, assume that $\Theta$ is symmetrizable. If a nontrivial linear combination of $a, b$ is 0 , then we are done, since of course 0 is a fixed point of $C$.

Suppose then that the system $\{a, b\}$ is linearly independent. By definition, there exist two unitary matrices $U_{1}$ and $U_{2}$ such that $U_{1} \Theta(z) U_{2}$ is symmetric for all $z \in \mathbb{D}$. Write

$$
U_{1}=\left(\begin{array}{cc}
\mu & -\bar{\lambda} \\
\lambda & \bar{\mu}
\end{array}\right), \quad U_{2}=\left(\begin{array}{cc}
\theta & -\bar{\gamma} \\
\gamma & \bar{\theta}
\end{array}\right)
$$

with $|\mu|^{2}+|\lambda|^{2}=1$ and $|\theta|^{2}+|\gamma|^{2}=1$. Straightforward computations show that

$$
U_{1} \Theta(z) U_{2}=\left(\begin{array}{cc}
* & X \\
Y & *
\end{array}\right)
$$

with $X=-\mu(\bar{\gamma} a+\bar{\theta} b)-\bar{\lambda} C(-\gamma b+\theta a)$ and $Y=\lambda(\theta a-\gamma b)+\bar{\mu} C(\bar{\theta} b+\bar{\gamma} a)$. Then the symmetry of the matrix is equivalent to

$$
-(\mu \bar{\gamma}+\lambda \theta) a-(\mu \bar{\theta}-\lambda \gamma) b=C((\mu \bar{\gamma}+\lambda \theta) a+(\mu \bar{\theta}-\lambda \gamma) b)
$$


If we put $u:=(\mu \bar{\gamma}+\lambda \theta) a+(\mu \bar{\theta}-\lambda \gamma) b$, then it follows that $C(u)=-u$, that is, $C(i u)=i u$, and $i u$ is a fixed point of $C$. To conclude the proof, we need to show that $(\mu \bar{\gamma}+\lambda \theta, \mu \bar{\theta}-\lambda \gamma) \neq(0,0)$.

Suppose then that

$$
\mu \bar{\gamma}+\lambda \theta=\mu \bar{\theta}-\lambda \gamma=0
$$

If we multiply $\mu \bar{\gamma}=-\lambda \theta$ by $\bar{\theta}$ and $\mu \bar{\theta}=\lambda \gamma$ by $\bar{\gamma}$, and subtract, we obtain $\lambda\left(|\theta|^{2}+\right.$ $\left.|\gamma|^{2}\right)=0$. But $|\theta|^{2}+|\gamma|^{2}=1$, so $\lambda=0$, whence $|\mu|^{2}+|\lambda|^{2}=1$ yields $|\mu|=1$. Then (4.2) implies $\gamma=\theta=0$ : a contradiction.

Remark 4.4. Note that the fixed points of a conjugation $C$ can easily be described by using Lemma2.1(i). They form the real vector space of all elements which have real Fourier coefficients with respect to a $C$-real orthonormal basis.

Remark 4.5. A closely related question would be to describe all symmetric $2 \times 2$ matrix valued analytic contractive inner functions $\Theta(z)$. This can be done along the lines of the solution of the Darlington sythesis problem in [3, Section 5], as follows. We first fix $\operatorname{det} \Theta$, which will be a nonconstant scalar inner function $\phi \in H^{\infty}$. Then we take a function $b \in H^{2} \ominus z \phi H^{2}$, such that $C b=b(C$ the conjugation $f \mapsto \phi \bar{f}$ on $H^{2} \ominus z \phi H^{2}$ ). If $b$ is inner, then $b^{2}=\phi$, and

$$
\Theta(z)=\left(\begin{array}{cc}
0 & i b(z) \\
i b(z) & 0
\end{array}\right)
$$

If $b$ is not inner, then we take $a \in H^{2} \ominus z \phi H^{2}$, such that $|a|^{2}+|b|^{2}=1$ (such $a$ 's exist by [3, Proposition 5.2]). Then

$$
\Theta(z)=\left(\begin{array}{cc}
a(z) & i b(z) \\
i b(z) & C(a)(z)
\end{array}\right)
$$

In [5, 8.2] one further discusses the parametrization of all rational solutions of a Darlington synthesis. Similarly, one could describe all rational symmetric $2 \times 2$ matrix valued analytic contractive inner functions $\Theta(z)$.

However, our interest is in rather complex symmetric contractions, and the characteristic function is only a method of studying them. If we want to parametrize, up to unitary equivalence, all complex symmetric contractions with defect indices 2, then we also need to determine when two symmetric characteristic functions coincide. This problem does not seem to have a neat solution.

\section{An example}

Consider two nonconstant scalar inner functions $u, v \in H^{\infty}$, and let $\mathbf{T}_{u}, \mathbf{T}_{v}$ be the corresponding model operators (the compressed shifts in the terminology of [3]). The corresponding model spaces are $\mathfrak{K}_{u}=H^{2} \ominus u H^{2}$ and $\mathfrak{K}_{v}=H^{2} \ominus v H^{2}$. As noted above, $\mathbf{T}_{u}$ and $\mathbf{T}_{v}$ are completely nonunitary contractions with characteristic functions $u$ and $v$ respectively. Their defect spaces are 1-dimensional, and it follows from Corollary 3.2 that they are both complex symmetric.

We will discuss the contractions of the form

$$
T=\left(\begin{array}{cc}
\mathbf{T}_{u} & X \\
0 & \mathbf{T}_{v}
\end{array}\right) ;
$$

thus $T \in \mathcal{L}(\mathcal{H})$, with $\mathcal{H}=\mathfrak{K}_{u} \oplus \mathfrak{K}_{v}$. The next lemma gathers some facts about this operator. 
Lemma 5.1. Suppose $T \in \mathcal{L}\left(\mathfrak{K}_{u} \oplus \mathfrak{K}_{v}\right)$ is a contraction. Then:

(i) $X=D_{\mathbf{T}_{u}^{*}} Y D_{\mathbf{T}_{v}}$, with $Y: \mathcal{D}_{\mathbf{T}_{v}} \rightarrow \mathcal{D}_{\mathbf{T}_{u}^{*}}$ a contraction.

(ii) $\partial_{T}=\partial_{T^{*}}=1$ if $\|Y\|=1$, and $\partial_{T}=\partial_{T^{*}}=2$ otherwise.

(iii) $T \in C_{00}$.

Note that, since $\operatorname{dim} \mathcal{D}_{\mathbf{T}_{v}}=\operatorname{dim} \mathcal{D}_{\mathbf{T}_{u}^{*}}=1, Y$ can actually be identified with a complex number of modulus not larger than 1 .

Proof. The general form of the entries of a $2 \times 2$ contraction, as described, for instance, in [1, Theorem 1.3] or [2, IV.3], applied to the case when one of the entries is null, immediately yields (i), as well as an identification of $\mathcal{D}_{T}$ with $\mathcal{D}_{\mathbf{T}_{v}^{*}} \oplus \mathcal{D}_{Y}$, and of $\mathcal{D}_{T^{*}}$ with $\mathcal{D}_{\mathbf{T}_{v}} \oplus \mathcal{D}_{Y^{*}}$, whence (ii) follows.

Finally, (iii) is an instance of a more general fact: if $T=\left(\begin{array}{cc}T_{1} & X \\ 0 & T_{2}\end{array}\right)$ is a contraction, then $T_{i} \in C_{0}$. implies $T \in C_{0}$. Indeed, take $\epsilon>0$, and a vector $x=x_{1} \oplus x_{2}$. First choose $k$ such that $\left\|T_{2}^{k} x_{2}\right\|<\epsilon$. If $T^{k}\left(0 \oplus x_{2}\right)=x_{1}^{\prime} \oplus T_{2}^{k} x_{2}$, take $k^{\prime}$ such that $\left\|T_{1}^{k^{\prime}}\left(x_{1}^{\prime} \oplus T_{1}^{k} x_{1}\right)\right\|<\epsilon$. Then

$$
\begin{aligned}
\left\|T^{k+k^{\prime}} x\right\| & =\left\|T^{k+k^{\prime}}\left(x_{1} \oplus 0\right)+T^{k+k^{\prime}}\left(0 \oplus x_{2}\right)\right\|=\left\|\left(T_{1}^{k+k^{\prime}} x_{1} \oplus 0\right)+T^{k^{\prime}}\left(x_{1}^{\prime} \oplus T_{2}^{k} x_{2}\right)\right\| \\
& \leq\left\|\left(T_{1}^{k^{\prime}}\left(T_{1}^{k} x_{1}+x_{1}^{\prime}\right) \oplus 0\right)\right\|+\left\|T^{k^{\prime}}\left(0 \oplus T_{2}^{k} x_{2}\right)\right\| \leq \epsilon+\epsilon=2 \epsilon .
\end{aligned}
$$

Since in our case $T_{1}=\mathbf{T}_{u}$ and $T_{2}=\mathbf{T}_{v}$ are both of class $C_{00}$, the result follows.

The next theorem determines when is $T$ complex symmetric.

Theorem 5.2. $T$ is complex symmetric precisely in the following cases:

(i) $Y=0$;

(ii) $\|Y\|=1$;

(iii) $0<\|Y\|<1$ and there exist $\lambda \in \mathbb{D}$ and $\mu \in \mathbb{T}$ such that $v=\mu b_{\lambda}(u)$, where $b_{\lambda}$ denotes the elementary Blaschke factor defined by

$$
b_{\lambda}(z)=\frac{\lambda-z}{1-\bar{\lambda} z}
$$

Proof. If $Y=0$, then $T=\mathbf{T}_{u} \oplus \mathbf{T}_{v}$, and it is therefore complex symmetric as the direct sum of two complex symmetric operators. If $\|Y\|=1$, then $T$ has defect indices 1 by Lemma 5.1, and is therefore complex symmetric by Corollary 3.2. (One can then easily see, using (5.2) below, that $\Theta_{T}$ coincides with the scalar function uv.) We can thus suppose in the sequel that $0<\|Y\|<1$, and $\partial_{T}=\partial_{T^{*}}=2$.

Since we intend to apply Theorem 3.1, we have to determine the characteristic function of $T$. This can be calculated directly, but in order to avoid some tedious computations, we prefer to use the theory of invariant subspaces of contractions and factorizations of the characteristic function, as developed in [6, Chapter VII].

First, note that $T \in C_{00}$ implies $\Theta_{T}$ inner. Since $\mathfrak{K}_{u}$ is an invariant subspace for $T$, it follows from Theorem VII.1.1 and Proposition VII.2.1 from [6] that one can factorize

$$
\Theta_{T}(z)=\Theta_{2}(z) \Theta_{1}(z)
$$

into two analytic inner functions, and that the characteristic functions of $\mathbf{T}_{u}$ and $\mathbf{T}_{v}$, that is, $u$ and $v$, are equal to the pure parts of $\Theta_{1}$ and $\Theta_{2}$. Also, $\Theta_{1}$ and $\Theta_{2}$ both being inner, the dimensions of their range spaces are both equal to the dimension of the range of $\Theta_{T}$. 
It then follows that $\Theta_{1}$ and $\Theta_{2}$ must be $2 \times 2$ matrix valued inner functions, and their pure parts are $u$ and $v$ respectively. They coincide therefore with $\left(\begin{array}{ll}1 & 0 \\ 0 & u\end{array}\right)$ and $\left(\begin{array}{ll}1 & 0 \\ 0 & v\end{array}\right)$ respectively. According to (5.2), we have $2 \times 2$ unitary matrices $U_{1}, U_{2}, V_{1}, V_{2}$ such that

$$
\Theta_{T}=U_{1}\left(\begin{array}{ll}
1 & 0 \\
0 & v
\end{array}\right) U_{2} V_{1}\left(\begin{array}{ll}
1 & 0 \\
0 & u
\end{array}\right) V_{2}
$$

If we write

$$
U_{2} V_{1}=\left(\begin{array}{cc}
\alpha & -\beta \\
\bar{\beta} & \bar{\alpha}
\end{array}\right)
$$

with $\alpha, \beta$ complex numbers satisfying $|\alpha|^{2}+|\beta|^{2}=1$, it follows that the characteristic function $\Theta_{T}$ coincides with the inner function

$$
\Theta(z)=\left(\begin{array}{cc}
1 & 0 \\
0 & v
\end{array}\right)\left(\begin{array}{cc}
\alpha & -\beta \\
\bar{\beta} & \bar{\alpha}
\end{array}\right)\left(\begin{array}{cc}
1 & 0 \\
0 & u
\end{array}\right)=\left(\begin{array}{cc}
\alpha & -\beta u(z) \\
\bar{\beta} v(z) & \bar{\alpha} u(z) v(z)
\end{array}\right) .
$$

Note that condition $0<\|Y\|<1$ implies both $\alpha$ and $\beta$ different from 0 .

We now apply Theorem 4.3 in order to determine when $\Theta$, as given by (5.3), is symmetrizable. Since $\operatorname{det} \Theta=u v$, this happens if and only if a linear combination of $\alpha$ and $\beta u$, not having both coefficients null, belongs to the fixed points of the conjugation $C$ on $\mathfrak{K}_{z u v}$ given by $C(f)=u v \bar{f}$.

If this is the case, and we write the combination as $g=s+t u, s, t \in \mathbb{C}$ (and $s, t$ are not both null, which implies also $g \neq 0$ ), then

$$
C(g)=g \Leftrightarrow v(\bar{s} u+\bar{t})=s+t u,
$$

and thus

$$
v=\frac{s+t u}{\bar{s} u+\bar{t}}
$$

We must have $t \neq 0$, since otherwise $u v$ is constant, which is not possible. So we can write

$$
v=\frac{t}{\bar{t}} \frac{\frac{s}{t}+u}{1+\frac{\bar{s}}{\bar{t}} u}
$$

But now if $|s|=|t|$, then $v=\frac{t}{\bar{s}}$ which is impossible. If $|s|>|t|$, then we see that $v$ is at the same time analytic and coanalytic; whence $v$ is constant - again a contradiction. So the only possibility is $|s|<|t|$. If we put $\lambda=-\frac{s}{t}$ and $\mu=-\frac{t}{\bar{t}}$ we get the desired conclusion that $v=\mu b_{\lambda}(u)$.

Conversely, suppose $v=\mu b_{\lambda}(u)$ with $|\lambda|<1$ and $|\mu|=1$. Write $\mu=-\frac{\zeta}{\zeta}$, with $\zeta \neq 0$. Then

$$
v=\frac{\zeta u-\lambda \zeta}{\bar{\zeta}-\bar{\zeta} \lambda u}
$$

and if we define $s:=-\lambda \zeta$ and $t:=\zeta$, then

$$
v(\bar{s} u+\bar{t})=v(-\overline{\lambda \zeta} u+\bar{\zeta})=\zeta u-\lambda \zeta=s+t u,
$$

which implies by (5.4) that $C(g)=g$, with $g:=s+t u$. Since $t \neq 0$, we may apply Theorem 4.3 to conclude that $\Theta$ is symmetrizable.

We have thus proved that in the case $0<\|Y\|<1, \Theta_{T}$ is symmetrizable if and only if $v=\mu b_{\lambda}(u)$ with $|\lambda|<1$ and $|\mu|=1$. Now applying Theorem 3.1 ends the proof. 
Using Theorem 5.2, it is easy to construct examples of complex symmetric as well as noncomplex symmetric operators with defect indices 2 .

It is not surprising that the condition obtained depends on the norm of $\|Y\|$ (or, rather, its modulus), and not on $Y$ itself. Indeed, with a little effort one can show that all operators $T$ with $u, v$ and $\|Y\|$ fixed are unitarily equivalent.

\section{REFERENCES}

1. Gr. Arsene and A. Gheondea, Completing matrix contractions, J. Operator Theory 7 (1982), 179-189. MR0650203(83i:47010)

2. C. Foias and A.E. Frazho, The Commutant Lifting Approach to Interpolation Problems, Birkhäuser Verlag, Basel, 1990. MR.1120546 (92k:47033)

3. S.R. Garcia, Conjugation, the backward shift, and Toeplitz kernels, J. Operator Theory 54:2 (2005), 239-250. MR2186351 (2006g:30055)

4. Conjugation and Clark operators, Contemporary Mathematics, vol. 393, Amer. Math. Soc., Providence, RI, 2006. MR2198373

5. S.R. Garcia and M. Putinar, Complex symmetric operators and applications, Trans. Amer. Math. Soc. 358:3 (2006), 1285-1315. MR2187654 (2006j:47036)

6. B. Sz-Nagy and C. Foias, Harmonic Analysis of Operators on Hilbert Space, North Holland, New York, 1970. MR0275190(43:947)

Institut Camille Jordan, UfR de Mathématiques, Université Claude Bernard Lyon I, 69622 Villeurbanne Cedex, France

E-mail address: chevrot@math.univ-lyon1.fr

Institut Camille Jordan, UfR de Mathématiques, Université Claude Bernard Lyon I, 69622 Villeurbanne Cedex, France

E-mail address: fricain@math.univ-lyon1.fr

Institute of Mathematics of the Romanian Academy, P.O. Box 1-764, Bucharest 014700, ROMANIA

E-mail address: Dan.Timotin@imar.ro 